\title{
Análise e monitoramento das taxas de cesárea no Brasil segundo a classificação de
}

\section{Robson}

\author{
Analysis and monitoring of cesárea fees in Brazil according to the Robson classification \\ Análisis y seguimiento de las tarifas de cesárea en Brasil según la clasificación de Robson
}

Rayssa Stéfani Sousa Alves ORCID: https://orcid.org/0000-0002-9666-675X Pontifícia Universidade Católica de Goiás, Brasil E-mail: rayssastefani02@gmail.com

Mariana Pereira Barbosa Silva ORCID: https://orcid.org/0000-0003-0852-8099 Universidade Estadual do Piauí, Brasil

E-mail: marianapbsilvaa@gmail.com Airton César Leite ORCID: https://orcid.org/0000-0001-7184-8488 Centro Universitário Santo Agostinho, Brasil E-mail: ainton.cesar2014@gmail.com

Elielson Rodrigues da Silva

ORCID: https://orcid.org/0000-0002-9628-1809 Centro Universitário do Rio São Francisco, Brasil

E-mail: elielsonfasvipa@gmail.com

Jaqueline Araújo Cunha

ORCID: https://orcid.org/0000-0002-3577-786X Universidade Paulista, Brasil

E-mail: jaquelinearaujocunha@gmail.com

Mayara Martins de Carvalho

ORCID: https://orcid.org/0000-0003-3889-8525

Universidade Federal do Piauí, Brasil

E-mail: mayaramartinsc@ hotmail.com

Bruno Lopes Pereira

ORCID: https://orcid.org/0000-0003-2324-4721

Instituto Educacional Santo Agostinho, Brasil

E-mail: brunoloperz192@gmail.com

Lucília da Costa Silva

ORCID: https://orcid.org/0000-0001-9386-5684

Centro Universitário Santo Agostinho, Brasil

E-mail: luciliafiso@outlook.com

Alane Dionizio Passos

ORCID: https://orcid.org/0000-0002-1373-5626

Centro Universitário Maurício de Nassau, Brasil

E-mail: alanepassos02@gmail.com

Mayra Paula Sales Morais

ORCID: https://orcid.org/0000-0003-1561-7372

Universidade Regional do Cariri, Brasil

E-mail: mayramorais_enfer@outlook.com

Kelly Savana Minaré Baldo Sucupira

ORCID: https://orcid.org/0000-0003-1932-9458

Universidade Federal do Triângulo Mineiro, Brasil

E-mail: kellyminare@gmail.com

Angelica Taciana Sisconetto

ORCID: https://orcid.org/0000-0001-6396-7372

Universidade Federal do Triângulo Mineiro, Brasil

E-mail: angelicasisconeto@hotmail.com

Vanessa Cristina Regis da Silva

ORCID: https://orcid.org/0000-0001-8415-5779

Universidade Federal do Triângulo Mineiro, Brasil E-mail: vanessacrisfisio@hotmail.com

Joelma Maria dos Santos da Silva Apolinário

ORCID: https://orcid.org/0000-0001-9521-9432 Faculdade Maurício de Nassau, Brasil E-mail: jo.silva00@hotmail.com 


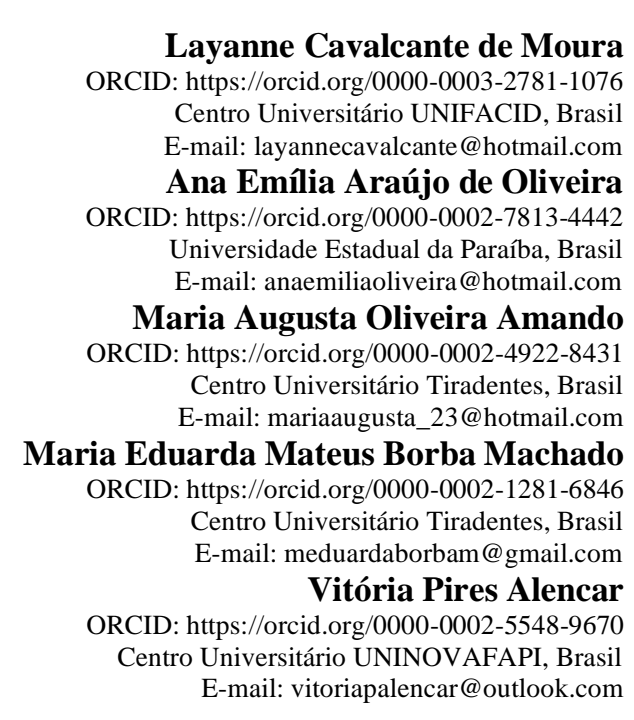

Layanne Cavalcante de Moura

E-mail: layannecavalcante@hotmail.com

Ana Emília Araújo de Oliveira

E-mail: anaemiliaoliveira@hotmail.com

Maria Augusta Oliveira Amando E-mail: mariaaugusta_23@hotmail.com

Maria Eduarda Mateus Borba Machado Centro Universitário Tiradentes, Brasil

Vitordaborbam@gmail.com

RCID: https://orcid.org/0000-0002-5548-9670 E-mail: vitoriapalencar@outlook.com

\section{Resumo}

Objetivo: Discorrer o que a literatura apresenta sobre os fatores de riscos obstétricos das mulheres submetidas à cesárea, segundo a Classificação de Robson. Método: Trata-se, de uma revisão bibliográfica do método revisão integrativa da literatura, com abordagem qualitativa, realizado entre setembro de 2020 a fevereiro de 2021, por meio da busca de artigos indexados nas seguintes bases de dados: Scielo (Scientific Eletronic Library Online), BVS (Biblioteca Virtual em Saúde), Google Scholar, PubMed (National Center for Biotechnology Information), Lilacs (Literatura Latino-Americana e do Caribe em Ciências da Saúde), Revistas de Enfermagem. Os critérios de inclusão para a seleção dos conteúdos foram, artigos na linguagem portuguesa, inglesa e espanhola. Publicados nos referidos bancos de dados compreendendo os anos de 2011 a 2021. Como critérios de exclusão, não foram considerados artigos mediante a recompensação monetária, incompletos, duplicados e não convergentes com este estudo. Resultados: Este estudo ressalta que, a cesárea é um procedimento que possui mais risco para as mulheres, quando comparado ao parto normal, posto isso, em decorrência dos fatores de riscos para a mulher e recém nascidos, a cesariana realizada sem as existentes indicações médicas tem sido alvo de estudos em todo o mundo. Sendo assim, é necessário identificar a real indicação do parto cesáreo sem que isto ocorra somente por comodidade médica. Conclusão: Evidenciou-se no estudo, que é a utilização da Classificação de Robson mostra-se extremamente útil e pode auxiliar em programas para monitorar as indicações de cesáreas, com probabilidade de redução das taxas e mortalidade materna.

Palavras-chave: Cesárea; Parto obstétrico; Classificação; Gravidez.

\section{Abstract}

Objective: To discuss what the literature presents about the obstetric risk factors of women undergoing cesarean section, according to the Robson Classification. Method: This is a bibliographic review of the integrative literature review method, with a qualitative approach, carried out between September 2020 and February 2021, through the search for articles indexed in the following databases: Scielo (Scientific Eletronic Library Online), VHL (Virtual Health Library), Google Scholar, PubMed (National Center for Biotechnology Information), Lilacs (Latin American and Caribbean Literature in Health Sciences), Nursing Journals. The inclusion criteria for the selection of content were articles in Portuguese, English and Spanish. Published in the aforementioned databases covering the years 2011 to 2021. As exclusion criteria, they were not considered articles through monetary reward, incomplete, duplicated and not converging with this study. Results: This study emphasizes that, cesarean section is a procedure that has a higher risk for women, when compared to normal delivery, since, due to the risk factors for women and newborns, the cesarean section performed without the existing medical indications has been the subject of studies all over the world. Therefore, it is necessary to identify the real indication for cesarean delivery without this occurring only for medical convenience. Conclusion: It was evidenced in the study, that the use of the Robson Classification is extremely useful and can assist in programs to monitor the indications for cesarean sections, with probability of reducing rates and maternal mortality.

Keywords: Cesarean section; Obstetric delivery; Classification; Pregnancy.

\section{Resumen}

Objetivo: discutir lo que presenta la literatura sobre los factores de riesgo obstétrico de las mujeres sometidas a cesárea, según la Clasificación de Robson. Método: Se trata de una revisión bibliográfica del método de revisión integradora de la literatura, con enfoque cualitativo, realizada entre septiembre de 2020 y febrero de 2021, mediante la búsqueda de artículos indexados en las siguientes bases de datos: Scielo (Scientific Eletronic Library Online), BVS (Virtual Health Library), Google Scholar, PubMed (Centro Nacional de Información Biotecnológica), Lilacs (Literatura Latinoamericana y del Caribe en Ciencias de la Salud), Revistas de Enfermería. Los criterios de inclusión para la selección de contenidos fueron artículos en portugués, inglés y español. Publicado en las citadas bases de datos que cubren los años 2011 a 2021. Como criterio de exclusión, no se consideraron artículos por recompensa monetaria, incompletos, duplicados y no convergentes con este estudio. Resultados: Este estudio destaca que, la 
cesárea es un procedimiento que presenta un mayor riesgo para la mujer, en comparación con el parto normal, ya que, debido a los factores de riesgo para la mujer y el recién nacido, la cesárea realizada sin las indicaciones médicas existentes ha sido la sujeto de estudios en todo el mundo. Por lo tanto, es necesario identificar la indicación real de parto por cesárea sin que esto ocurra solo por conveniencia médica. Conclusión: Se evidenció en el estudio, que el uso de la Clasificación de Robson es de suma utilidad y puede ayudar en programas de seguimiento de las indicaciones de cesáreas, con probabilidad de reducción de tasas y mortalidad materna.

Palabras clave: Cesárea; Parto obstétrico; Clasificación; Embarazo.

\section{Introdução}

O parto consiste no desfecho do processo gestacional, podendo acontecer por duas vias: via baixa, parto vaginal e via alta, cesárea. A operação cesariana é um dos procedimentos cirúrgicos mais antigos e com maior frequência no mundo. O parto realizado por meio da cesárea consiste numa incisão cirúrgica abdominal e em uma incisão uterina para a retirada do feto (Yoshhizaki, 2016).

Desde a Década de 1970 verifica-se um aumento nas taxas de cesáreas, o que tem gerado um alerta na comunidade científica. Em 1985, a Organização Mundial de Saúde (OMS), declarou que o percentual ideal de cesárea em um país deve ser entre $10 \%$ e $15 \%$ dos partos. Entretanto, as taxas de cesárea vêm aumentando nas últimas décadas, tanto em países desenvolvidos como naqueles em desenvolvimento (OMS, 2015; Yoshhizaki, 2016).

Diante deste cenário, e como ao longo das últimas décadas surgiram mais informações sobre os benefícios e riscos da cesárea e melhoras nos cuidados obstétricos, o órgão realizou uma revisão sistemática de estudos para reavaliar essa recomendação (OMS, 2015).

Em 2015, a OMS, publicou uma nova Declaração sobre Taxas de Cesárea, relatando que as cesáreas são eficientes para salvar vidas de mães e crianças, quando bem indicadas e feitas em ambiente seguro, todavia adverte dos riscos (OMS, 2015).

Uma revisão de literatura realizada por Santos e Araújo (2016), elencou os principais documentos, voltados para a saúde da mulher e do recém-nascido, que surgiram no Brasil. No final do Século XX destaca-se: o Programa Nacional de Saúde MaternoInfantil (PNSMI) em 1974; o Programa de Assistência Integral à Saúde da Mulher (PAISM) em 1984; a Portaria ${ }^{\circ}$.1.016 sobre as Normas Básicas para Implantação do Sistema de Alojamento Conjunto de 1993; a Portaria no 985 , de 5 de agosto de 1999 que determinou o Centro de Parto Normal-CPN, para o atendimento à mulher no período gravídico-puerperal (Santos; Araújo, 2016).

No Século XXI foram desenvolvidos inúmeros documentos com enfoque na saúde da mulher. Em 2000, por meio da Portaria $n^{\circ}$ 569/2000 criou-se o Programa de Humanização no Pré-Natal e Nascimento e, no mesmo ano, o Projeto Maternidade Segura. Em 2004 surgiu o Pacto Nacional pela Redução da Mortalidade Materna e Neonatal, e em 2005 a Lei do Acompanhante (Santos; Araújo, 2016).

A Rede Amamenta Brasil surgiu em 2008, por meio da Portaria n 2.799/2008, e a Rede Cegonha através das Portarias $n^{\circ} 1.459 / 2011$ e n⿳0 650/2011. O documento mais recente surgiu em 2016, que é as Diretrizes de Atenção à Gestante: a Operação Cesariana regulamentada pela Portaria no 306/2016 (Santos; Araújo, 2016).

Apesar do surgimento de tantos documentos nas últimas décadas, o Brasil teve aumento das taxas de cesárea. Uma contradição ao que deveria ocorrer e na contramão da recomendação da OMS. Segundo o MS, por mais que as complicações relacionadas à cesariana sejam pouco frequentes, com a alta taxa de realização desse procedimento, há um aumento significativo do número de complicações cirúrgicas graves (Brasil, 2016, p.18).

Em relação ao aspecto neonatal, a cesariana desnecessária contribui “para a prematuridade tardia iatrogênica, a ocorrência de desconforto respiratório e a internação em unidade de terapia intensiva neonatal", pelo fato de ser realizada no pré termo, ou seja, ao redor da $37^{\mathrm{a}}$ semana de gestação (Brasil, 2016, p.18).

O Boletim Epidemiológico da Secretária de Vigilância em Saúde (SVS) relata dados sobre a Mortalidade Materna. No período compreendido entre 1996 a 2018, ocorreram 38.919 óbitos maternos, dos quais 67\% foram por causas obstétricas diretas. As principais causas obstétricas diretas se destacaram a hipertensão, hemorragia, infecção puerperal e aborto (Brasil, 2020). 
Diante da temática em discussão, surge como questão norteadora do estudo: "O que a literatura apresenta acerca do perfil obstétrico de mulheres submetidas à cesariana segundo a Classificação de Robson"? Assim, o objetivo deste estudo consiste em realizar uma revisão da literatura compreendendo os riscos obstétricos de mulheres submetidas à cesárea, segundo a Classificação de Robson.

Desse modo, o estudo trará contribuições importantes para a comunidade científica e para a sociedade sobre a Classificação de Robson, este estudo poderá auxiliar os profissionais da saúde a obterem conhecimento sobre o perfil das mulheres que foram submetidas a cesarianas, e leva-los a questionar quanto a indicação do procedimento, visando favorecer a realização de ações de educação permanente para os profissionais da saúde no âmbito obstétrico, atentando - se, para o perfil obstétrico de mulheres, para indicação adequada de cesáreas segundo a Classificação de Robson.

\section{Metodologia}

Trata-se, de uma revisão bibliográfica do método revisão integrativa da literatura, com abordagem qualitativa, realizado entre setembro de 2020 a fevereiro de 2021. A revisão configura-se, portanto, como um tipo de revisão da literatura que reúne achados de estudos desenvolvidos mediante diferentes metodologias, permitindo aos pesquisadores sintetizar resultados sem ferir a filiação epistemológica dos estudos empíricos incluídos (Soares et al., 2014).

A revisão integrativa é um método que tem como finalidade reunir e sintetizar resultados de pesquisas sobre um delimitado tema ou questão, de maneira sistemática e ordenada e abrangente, contribuindo para o aprofundamento do conhecimento do tema investigado (Ferenhof \& Fernandes, 2016), ou seja, a revisão de literatura é o processo de busca, análise e descrição de um corpo do conhecimento em busca de resposta a uma pergunta específica (UNESP, 2015).

O levantamento de conteúdo foi realizado por meio da busca de artigos indexados nas seguintes bases de dados: Scielo (Scientific Eletronic Library Online), BVS (Biblioteca Virtual em Saúde), Google Scholar, PubMed (National Center for Biotechnology Information), Lilacs (Literatura Latino-Americana e do Caribe em Ciências da Saúde), Revistas de Enfermagem.

Os critérios de inclusão para a seleção dos conteúdos foram, artigos na linguagem portuguesa, inglesa e espanhola. Publicados nos referidos bancos de dados compreendendo os anos de 2011 a 2021.

Os critérios de exclusão foram, artigos que não tivessem relevância com a temática, materiais duplicados, incompletos, resumos, resenhas, debates, relato de caso, relato de experiência, publicados em anais de eventos e materiais indisponíveis na íntegra. 


\section{Resultados e Discussão}

Para o levantamento dos dados referente ao monitoramento das taxas de cesárea segundo a classificação de Robson, foram encontradas 96 produções científicas com os descritores utilizados, e 8 estudos referentes à entidades de saúde, portanto, apenas 44 permaneceram após a exclusão dos registros duplicados. 38 estudos foram triados, destes, 14 foram excluídos, restando 24 artigos para avaliação compreendendo os critérios de elegibilidade, destes, 13 estudos completos foram excluídos por não atender aos critérios para composição e análise deste estudo, restando então, 11 estudos para análise dos dados. O fluxograma com o detalhamento das etapas de pesquisa está apresentado a seguir na Figura 1.

Figura 1. Fluxograma de identificação e seleção dos artigos. 2021.

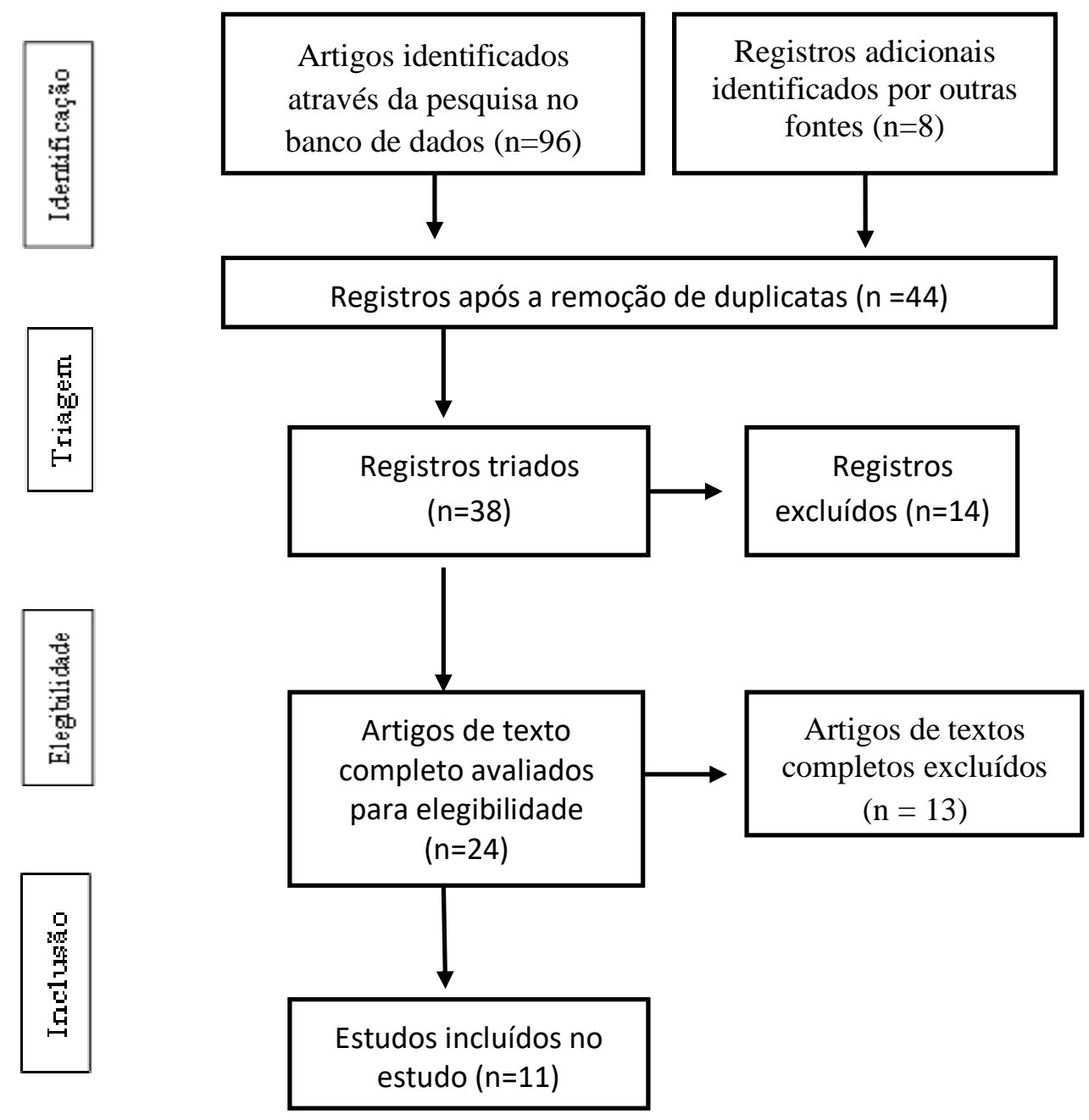

Fonte: Autores (2021)

Em decorrência dos fatores de riscos para a mulher, a cesariana realizada sem as existentes indicações obstétricas e neonatais tem sido alvo de estudos em todo o mundo, pois este estudo ressalta que, a cesárea é um procedimento que possui mais risco para as mulheres, quando comparado ao parto normal (Mascarello; Horta; Silveira, 2017).

Uma revisão sistemática e meta-análise realizada por Mascarello, Horta e Silveira (2017), identificou oito estudos que avaliaram a presença de complicações puerperais graves, comparados quanto a via de parto. A revisão conclui que, em relação à transfusão de sangue e hemorragia, as evidências disponíveis são controversas, e são de qualidade muito baixa, e baixa, respectivamente (Mascarello; Horta; Silveira, 2017). 
A revisão confirmou que, a presença de infecção pós-parto e a necessidade de internação em UTI, foi maior em partos cesáreos. O risco de hemorragia, histerectomia e transfusão de sangue, aparenta ser maior apenas nas cesáreas intraparto (após o início do trabalho de parto), considerando que o parto cesáreo é um procedimento que envolve risco de morte materna dez vezes maior quando comparado ao parto normal (Barba; Barifouse 2014).

E em decorrência dos fatores de riscos para a mulher e recém nascidos, a cesariana realizada sem as existentes indicações médicas tem sido alvo de estudos em todo o mundo. Sendo assim, é necessário identificar a real indicação do parto cesáreo sem que isto ocorra somente por comodidade médica (Mascarello; Horta; Silveira, 2017).

Nas últimas décadas, houve um aumento progressivo na taxa de partos por cesariana na maioria dos países, mas os fatores que motivam essa tendência não são completamente compreendidos. $\mathrm{O}$ aumento das taxas de cesárea é uma grande preocupação para a saúde pública e causa debates em todo o mundo devido a riscos maternos e perinatais (Patah; Malik, 2011).

A mortalidade materna é outro problema de saúde pública na maioria dos países. Em alguns casos está associada a realização de procedimentos invasivos, como a cesariana sem indicação. A análise das taxas de cesarianas é importante para que se possa identificar se as políticas públicas relacionadas a boas práticas na atenção obstétrica estão sendo eficazes, e para que se possa propor e implementar medidas efetivas para reduzir as taxas de cesárea (Mascarello; Horta; Silveira, 2017).

A realização desta proposta apresenta vários pontos positivos. O levantamento de dados sobre o perfil sociodemográfico e obstétrico das mulheres submetidas a cesárias, poderá auxiliar os gestores públicos no âmbito da saúde, na elaboração de medidas mais eficazes para a redução dessas taxas (Zanardo et al., 2017).

No Brasil, as taxas de cesárea aumentaram nas últimas décadas. Um estudo realizado pela fundação Fiocruz mostra esse aumento, de 1970 a 2010. Um gráfico elaborado pela fundação demonstra que, em 1970, o Brasil tinha uma taxa de cesáreas de aproximadamente 15\%. Essa taxa foi crescendo, chegando a um pouco mais de 50\% em 2010 (Leal; Gama, 2016).

Em uma Análise da Situação de Saúde do Brasil, realizada pelo Ministério da Saúde (MS) em 2018, verificou-se que as taxas de cesárea em 2014 foram de 57\%, e em 2015 de 55,5\%. No mesmo período que houve esse aumento dos partos cesáreos, como citado acima, houve a construção de importantes documentos incentivando a assistência ao parto vaginal e a prática da humanização (Brasil, 2018).

A Diretriz de Atenção à Gestante relata que a realização excessiva de cesarianas está relacionada a forma como a assistência ao parto é disposta no Brasil. A atenção voltada para a atuação individual dos profissionais, oposto à abordagem multidisciplinar e o trabalho em equipe. As características socioculturais, a qualidade do serviço ofertado ao nascimento, o modelo de assistência do pré-natal, que não possui o hábito de preparar adequadamente as mulheres para o parto (Brasil, 2016).

A taxa de cesariana é influenciada por vários fatores, que podem ser individuais, de ordem estrutural e sistêmica (modelo de atenção obstétrica), e a preferência dos profissionais de saúde e das gestantes. As características individuais estão relacionadas às características demográficas, clínicas e obstétricas. As características obstétricas destacam-se a paridade, cesariana prévia, apresentação fetal, modo de início do trabalho de parto entre outras características (Brasil, 2016).

A Classificação de Robson é realizada em 10 grupos, sendo utilizados 6 (seis) conceitos de obstetrícia para classificar as mulheres, quais sejam, paridade (nulípara, multípara); cesárea anterior (sim ou não); início do trabalho de parto (espontâneo, induzido, cesárea antes do trabalho de parto); idade gestacional (termo, pré-termo); apresentação fetal (cefálica, pélvica, transversa); número de fetos (único ou múltipla). Segundo a Fundação Oswaldo Cruz (2018), todas essas variáveis tornam a classificação totalmente inclusiva e mutualmente exclusiva, ou seja, todas as gestantes são incluídas em apenas um dos 10 grupos (WHO, 2017).

No Brasil, os dados utilizados na Classificação de Robson, encontram-se na Declaração de Nascido Vivo (DNV) É um documento padrão, de uso obrigatório em todo o território nacional. Na DNV foi adotado as variáveis utilizadas na Classificação de Robson, e outros importantes para vigilância epidemiológica (Brasil, 2011). 
Todos esses dados são inseridos no Sistema de Informações sobre Nascidos Vivos (SINASC), implantando pelo MS em 1990. Esse sistema tem como base a DNV, e seu objetivo é prover informações sobre as características dos nascimentos, que são basais para se estabelecer indicadores de saúde específicos (Brasil, 2011).

\section{Conclusão}

Evidenciou-se no estudo, que é a utilização da Classificação de Robson mostra-se extremamente útil e pode auxiliar em programas para monitorar as cesáreas, com probabilidade de redução das taxas observadas. Os gestores municipais e estaduais podem utilizar a potencialidade de dados contidos no SINASC para a formulação de indicadores epidemiológicos, que serviram de suporte ao planejamento de ações, atividades e programas voltados à gestão em saúde. Considerando que a cesariana é um procedimento cirúrgico de grande porte, com riscos para a mulher (anestesia, laceração acidental, infecção, hemorragia etc.) e o bebê (aumento da proporção de prematuridade, síndrome de angústia respiratória e necessidade de ressuscitação) decorrentes do próprio procedimento cirúrgico, somente deveria ser realizada com indicações claras, quando há riscos para a mulher e o bebê.

\section{Referências}

Brasil. (2011). Ministério da Saúde. Secretaria de Vigilância em Saúde. Departamento de Análise de Situação de Saúde. Manual de Instruções para o preenchimento da Declaração de Nascido Vivo. (Nota técnica).

Brasil. (2017). Ministério da Saúde Secretaria de Vigilância em Saúde Departamento de Vigilância de Doenças e Agravos Não Transmissíveis e Promoção de Saúde. Saúde Brasil 2017: uma análise da situação de saúde e os desafios para o alcance dos objetivos de desenvolvimento sustentável. [s.l: s.n.].

Brasil. (2016). Diretrizes de Atenção à Gestante: a operação Cesariana. Conitec, p. 101

Brasil. (2020). MINISTÉRIO DA SAÚDE. Boletim Epidemiológico: Mortalidade Materna no Brasil. Secretaria de Vigilância em Saúde. Coordenação-Geral de Informações e Análise Epidemiológica (CGIAE/DASNT/SVS), v. 51, n. 20, p. 21-27.

Fundação Oswaldo Cruz. (2018). Classificação de Robson. p. 32.

Leal, M. C.; \& Gama, S. G. N. (2016). Nascer no Brasil: Sumário Executivo Temático da Pesquisa.

Mascarello, K. C.; Horta, B. L.; Silveira, M. F. (2017). Complicações maternas e cesárea sem indicação: revisão sistemática e meta-análise. Revista de Saúde Pública, v. 51, p. 1-12.

OMS. Organização Mundial de Saúde. (2015). Declaração da OMS sobre Taxas de Cesáreas. Human Reproduction Programme, p. 1-8.

Robson, M. S. (2001). Can we reduce the caesarean section rate? Best Practice and Research: Clinical Obstetrics and Gynaecology, v. 15 , n. 1, p. 179-194.

Santos, H. F. L.; \& Araújo, M. M. (2016). Políticas De Humanização Ao Pré-Natal E Parto: Uma Revisão De Literatura Humanization the Policies Prenatal and Childbirth: a Literature Review. Revista Científica FacMais, v. 6, n. 2, p. 54-64.

World Health Organization. (2017). Robson Classification, Implementation manual. (Nota técnica). 